\title{
Echocardiographic Measurement of Pulmonary Arterial Pressure in Patients with Sarcoidosis
}

\author{
Ipek Candemir ${ }^{1}$, Ozlem Ozdemir Kumbasar ${ }^{2}$ \\ ${ }^{1}$ Ataturk Chest Diseases and Surgery Education and Research Hospital, Ankara, Turkey \\ ${ }^{2}$ Department of Chest Diseases, Ankara University Faculty of Medicine, Ankara, Turkey \\ Email: ipekcayli@yahoo.com, ozlemozdemir@yahoo.com
}

Received 2 February 2016; accepted 23 February 2016; published 26 February 2016

Copyright (C) 2016 by authors and Scientific Research Publishing Inc.

This work is licensed under the Creative Commons Attribution International License (CC BY). http://creativecommons.org/licenses/by/4.0/

(c) (i) Open Access

\begin{abstract}
Introduction: Pulmonary hypertension (PH) is a significant complication and is poor prognostic factor for sarcoidosis. We evaluated various tests of diagnosis and treatment as well as the associations between PH and value of six-minute walk test (6 MWT) in sarcoidosis patients. Methods: This study was conducted with fifty patients with sarcoidosis. We grouped patients according to echocardiography. Results: $\mathbf{P H}$ prevalence in sarcoidosis was found to be $10 \%$. In cases with likely PH, FEV1\%, FVC\%, MMF\% was found to be lower. Although DLCO was less than $80 \%$ in the group of likely $\mathrm{PH}$ and over $80 \%$ in the other, there were no statistical differences between two groups. Mean value of FVC/DLCO ratio was $1.1 \pm 0.38$ in group of likely PH. There was no significant difference between groups. Mean distance of 6 MWT was $464 \pm 105 \mathrm{~m}$ in all cases. There were no statistical differences between two groups in 6 MWT. Significant desaturation with exercise was observed in cases with PH $(p=0.007)$. Conclusion: In all stages, patients who have longer disease duration and abnormal pulmonary function tests, should be examined about PH. Patients should undergo 6 MWT and presence of desaturation after 6 MWT, even without hypoxemia, should be looked after and dealt with accordingly.
\end{abstract}

\section{Keywords}

Sarcoidosis, PH, ECO, PFT, 6 MWT

\section{Introduction}

Sarcoidosis has unknown reason and is multisystemic granulomatous disease that affects mostly lung and lymph

How to cite this paper: Candemir, I. and Kumbasar, O.O. (2016) Echocardiographic Measurement of Pulmonary Arterial Pressure in Patients with Sarcoidosis. Open Journal of Respiratory Diseases, 6, 7-13. 
nodes. Sarcoidosis can be seen in anywhere in the world and can occur at any age, sex, race. In 2007, Turkish Society clinical problems study group reported the sarcoidosis incidence in Turkey as 4/100.000 per year [1]. According to lung effects, the most common symptoms are dyspnea, cough, and chest pain. Because it is a systemic disease, any symptoms can be observed. Pulmonary hypertension $(\mathrm{PH})$ is rare but significant complication of sarcoidosis and has been demonstrated to be significantly associated with increased mortality [2]-[4]. Causes of sarcodoisis associated PH have not been clarified yet but possible explanations include damage to the vascular bed due to lung parenchymal fibrosis, granuloma in the pulmonary blood vessels, compression of the pulmonary vessels by lymphadenopathy, systolic/diastolic dysfunction associated myocardial sarcoidosis, and hypoxic vasoconstriction. Obstructive sleep apnea is another possible cause of $\mathrm{PH}$, as it is observed quite frequently in sarcoidosis [5]-[8].

The symptoms of right ventricular dysfunction such as progressive dyspnea, cough, chest pain, tachycardia, and pretibial edema can also be seen in sarcoidosis associated PH. Echocardiography, one of the most helpful diagnostic methods that is both noninvasive and practical, is also useful in diagnosis of cardiac dyspnea [9]. No certain and recent incidence of sarcoidosis associated PH was reported in our country.

In this study, we aimed to investigate the presence and frequency of PH and relationship between pulmonary arterial pressure and sarcoidosis related parameters (symptom, radiological stage, pulmonary function test, 6minute walk test (6 MWT)) and value of 6 MWT in our patients with sarcoidosis.

\section{Methods}

Fifty patients, who were followed-up at sarcoidosis unit, were enrolled into this prospective study in December 2007-June 2009. The annual admission of the unit is about $150-200$ patients. All the patients gave written permission and Ethics Committee of Ankara University approved the study. The exclusion criteria were to have a relative or absolute contraindication for 6 MWT [10] (unstable angina in the last month, history of myocardial infarction, pulse rate at rest over 120/min, systolic blood pressure over $180 \mathrm{mmHg}$ and diastolic blood pressure over $100 \mathrm{mmHg}$ ) according to American Thorax Society (ATS) and pathologies such as mitral stenosis, congestive heart failure, systemic hypertension, connective tissue diseases that might affect pulmonary arterial pressure. Physically disabled patients were also excluded.

Age, duration of disease, affected systems, blood gas analysis, radiological stage, pulmonary function test (PFT). The single-breath diffusing capacity of carbonmonoxide test (DLCO), 6 MWT, BORG scale and saturation before and after $6 \mathrm{MWT}$ of all patients were recorded. Blood gas analysis was performed at rest on room air by COBAS B 121 (ROCHE, West Roxbury, and USA). 6 MWT was performed according to ATS criteria. Before and after 6 MWT, dyspnea sensation assessmenty with BORG and saturation were recorded. The cut off value has not been declared for morbidity and mortality of 6 WMT. So, we did not use any cut off value.

PH possibility assessed with echocardiography (Vivid 7, GE, Wingmed, and Milwaukee, USA). It allows calculation of the right ventricular systolic pressures (RVSP) from the maximal velocity of the tricuspid regurgitation jet together with an estimation of the right atrial pressure [11]. In the absence of tricuspid regurgitation, the diagnosis of PH should still be suspected when there are signs of right ventricular (RV) dysfunction, including RV dilatation, hypertrophy, and decreased systolic function as well as flattening or bowing of the interventricular septum [12]. PH possibility was assessed with echocardiographic criteria according to $2009 \mathrm{PH}$ guideline [13]. PH unlikely was defined as tricuspid regurgitation velocity $\leq 2.8 \mathrm{~m} / \mathrm{s}$, PA systolic pressure $\leq 36$ $\mathrm{mmHg}$, and no additional echocardiographic variables suggestive of $\mathrm{PH}$. PH possible was defined as tricuspid regurgitation velocity $\leq 2.8 \mathrm{~m} / \mathrm{s}$, pulmonary arterial (PA) systolic pressure $\leq 36 \mathrm{mmHg}$, but presence of additional echocardiographic variables suggestive of PH or tricuspid regurgitation velocity $2.9-3.4 \mathrm{~m} / \mathrm{s}$, PA systolic pressure $37-50 \mathrm{mmHg}$ with/without additional echocardiographic variables suggestive of $\mathrm{PH}$ and $\mathrm{PH}$ likely was defined as tricuspid regurgitation velocity $>3.4 \mathrm{~m} / \mathrm{s}$, PA systolic pressure $>50 \mathrm{mmHg}$, with/without additional echocardiographic variables suggestive of $\mathrm{PH}[13]$.

According to this guideline, the patients were divided into two groups: Group 1—patients with PH unlikely and Group 2-patients with PH possible, likely). The relationship between systolic pulmonary arterial pressure (PAP) and 6 MWT, PFT, DLCO, disease duration, stage, blood gas analysis, BORG and saturation (before and after test) and also these parameters between groups were analyzed. The correlation between 6 MWT and these parameters were also analyzed. 


\section{Statistical Analysis}

SPSS version 15 (SPSS, Inc., Chicago, Illinois) for Microsoft Windows (Microsoft Corporation, Redmond, Washington) was used for analysis. Data are expressed as mean $\pm \mathrm{SD}$ or as median, depending on whether the data were normally distributed. Chi-square tests were used for comparisons of categorical values. Mann-Whitney U test was used to compare variables across groups with abnormally distribution and unpaired t-test for normally distributed variables. For correlation analysis, Spearman correlation analysis was used. Statistical significance was set to a p-value $<0.05$.

\section{Results}

Mean age of the patients was $45 \pm 14$ year. Forty two of them (84\%) were female; $8(16 \%)$ were male. Half of the patients' symptom was dyspnea $(23 \%-43 \%)$. Nine $(18 \%)$ patients had cough; $6(12 \%)$ had chest and back pain; $5(10 \%)$ had arthralgia (without arthritis); $2(4 \%)$ had tachycardia; $8(16 \%)$ had fatigue; 13 (26\%) had another symptoms. Half of the patients were stage $2 ; 14(28 \%)$ were stage $1 ; 6(12 \%)$ were stage $3 ; 5(10 \%)$ were stage 4. $23(46 \%)$ patients had only pulmonary involvement; 27 (54\%) of patients had pulmonary with extrapulmonary involvement. The most common (14 patients (28\%)) extra pulmonary involvement was skin. Holter monitoring, Gallium 67 scintigraphy and cardiac magnetic resonance were performed in 5 patients who had tachycardia and/or extrasystoles in electrocardiogram. Cardiac involvement was not present in any of the patients. No patients had muscle skeletal system involvement. General characteristics and functional parameters of all patients are given in Table 1.

Mean duration of sarcoidosis was 3 years. Mean values of Forced expiratory volume in one second (FEV1), Forced vital capacity (FVC), DLCO, Diffusing capacity divided by the alveolar volume (DLCO/VA) of the predicted \% were normal. Mean 6 MWT distance was $464 \pm 105 \mathrm{~m}$ and after the test BORG scale depressed 1.1 unit. Mean value of systolic PAP was $29 \pm 9.5 \mathrm{mmHg}$. Patients were divided into two groups according to PH possibility (Group 1: PH unlikely; Group 2: possible and likely). Distribution of stages of the groups was given in Table 2. Disease duration of the groups was analyzed by using independent t-test. In group 1, the duration was

Table 1. General characteristics and functional parameters.

\begin{tabular}{cccccc}
\hline Age (year) & N & Minimum & Maximum & Mean & Std. Deviation \\
\hline Disease duration (year) & 50 & 21 & 73 & 45 & 14 \\
FEV1/ FVC (predicted\% ratio) & 50 & 1 & 14 & 3 & 1 \\
FEV1 (predicted\%) & 50 & 42 & 95 & 78 & 8.9 \\
FVC (predicted\%) & 50 & 36 & 143 & 89 & 22.7 \\
MMF (predicted\%) & 50 & 38 & 146 & 96.3 & 21.7 \\
DLCO (predicted\%) & 37 & 14 & 116 & 69.4 & 24 \\
DLCO/VA (predicted\% ratio) & 49 & 21 & 151 & 86.5 & 26 \\
FVC/DLCO (predicted\% ratio) & 49 & 15 & 124 & 91 & 20 \\
6 MWT (meter) & 49 & 0.69 & 3.76 & 1.2 & 0.47 \\
Before 6MWT sat. (\%) & 50 & 180 & 720 & 464 & 105 \\
After 6MWT sat. (\%) & 50 & 90 & 100 & 96 & 2.3 \\
Borg (Before 6 MWT) & 50 & 70 & 100 & 93 & 6.5 \\
Borg (After 6 MWT) & 50 & 0.0 & 4.0 & 0.5 & 0.87 \\
SPAP (mmHg) & 50 & 0.0 & 7.0 & 1.64 & 2 \\
pH & 50 & 20 & 75 & 29 & 9.5 \\
PCO (mmHg) & 44 & 7.37 & 7.53 & 7.42 & 34 \\
PaO (mmHg) & 44 & 26 & 42 & 74 & 3.6 \\
\hline
\end{tabular}

FEV1: Forced expiratory volume in one second; FVC: Forced vital capacity; MMF: Maximum mid-expiratory flow; DLCO: Single breath carbon monoxide diffusing capacity; DLCO/VA: Diffusing capacity divided by the alveolar volume; 6 MWT: Six-minute walking test; sat.: saturation; sPAP: Systolic pulmonary arterial pressure; $\mathrm{PCO}_{2}$ : Partial pressure of $\mathrm{CO}_{2} ; \mathrm{PaO}_{2}$ : Partial pressure of $\mathrm{O}_{2}$. 
$2.5 \pm 3.1$ years, in group 2 it was $7.6 \pm 6.6$ years $(p=0.004)$. The most common symptom was dyspnea in both groups. Extrapulmonary involvement was found in 24 patients (53\%) in group 1; 60\% (3 patients) in group 2 and pulmonary involvement was found in 21 patients $(46 \%)$ in group $1 ; 2$ patients $(40 \%)$ in group 2 . In group 2 , FEV1 $(p=0.005), \operatorname{FVC}(p=0.018), \operatorname{MMF}(p=0.013)$ of the predicted $\%$, saturation before 6 MWT $(p=0.024)$, saturation after the test $(p=0.011)$ and desaturation $(p=0.007)$ were observed to be significantly lower than group 1 (Table 3). The mean value of FVC/DLCO predicted\% ratio was $1.2 \pm 0.48$ in group $1 ; 1.1 \pm 0.38$ in group 2. There was no significant difference between groups.

Positive correlation was found between systolic PAP and age $(p=0.022, \mathrm{r}=0.347)$ and negative correlation between systolic PAP and FEV1 of the predicted $\%(p=0.034, \mathrm{r}=-0.401)$, saturation before 6 MWT $(p=0.045$, $\mathrm{r}=-0.444)$, after 6 MWT $(p=0.041, \mathrm{r}=-0.643)$, MMF of the predicted $\%(p=0.007, \mathrm{r}=-0.673)$. Borderline correlation between PAP and FVC\% $(p=0.051, \mathrm{r}=-0.129)$. No correlation found between PAP and $6 \mathrm{MWT}$, DLCO, DLCO/VA of the predicted\%. The significant correlation between 6 MWT and age $(p<0.001, \mathrm{r}=$ $-0.716)$, stage $(p=0.012, \mathrm{r}=-0.485)$, FEV1 $(p=0.033, \mathrm{r}=0.276)$, FVC $(p=0.029, \mathrm{r}=0.349)$, DLCO $(p=$ $0.012, \mathrm{r}=0.424)$ of the predicted $\%, \mathrm{PO}_{2}(p=0.020, \mathrm{r}=0.389)$.

\section{Discussion}

Sarcoidosis is a multinational disease which is mostly seen in females [14] as in our country [1]. In this study 42 patients (84\%) were female. It is reported that it is seen frequently in ages between $34-35$, and $1 / 3$ patients are over 50 year. The mean age of our patients was also 45 year and 20 patients $(40 \%)$ of them were over 50 .

Table 2. Distribution of the stages between groups.

\begin{tabular}{cccc} 
& Group 1: PH unlikely (n:45) & Group 2: PH possible, likely (n:5) & Total \\
\hline Stage 1 & 13 & 0 & 13 \\
Stage 2 & 24 & 2 & 26 \\
Stage 3 & 6 & 0 & 6 \\
Stage 4 & 2 & 3 & 5 \\
\hline
\end{tabular}

Table 3. Compared parameter between groups

\begin{tabular}{|c|c|c|c|c|c|}
\hline & \multicolumn{2}{|c|}{ Group 1} & \multicolumn{2}{|c|}{ Group 2} & \multirow{2}{*}{$\mathbf{p}$} \\
\hline & Mean \pm SD & Median (max, min) & Mean \pm SD & Median (max, min) & \\
\hline Age (year) & $44 \pm 14$ & $45(73,21)$ & $54 \pm 7$ & $52(67,49)$ & 0.11 \\
\hline FEV1 (predicted\%) & $92.5 \pm 20$ & $95(143,40)$ & $57 \pm 23$ & $51(93,36)$ & 0.005 \\
\hline FVC (predicted\%) & $98 \pm 22$ & $101(146,38)$ & $77 \pm 12$ & $75(98,65)$ & 0.018 \\
\hline MMF (predicted\%) & $72 \pm 21.3$ & $70(116,41)$ & $37.6 \pm 23.6$ & $70(72,14)$ & 0.013 \\
\hline DLCO (predicted\%) & $88 \pm 26$ & $83(151,21)$ & $71 \pm 23$ & $81(97,43)$ & 0.23 \\
\hline DLCO/VA (predicted\% ratio) & $91 \pm 20$ & $91(124,15)$ & $92 \pm 26$ & $92(118,50)$ & 0.69 \\
\hline FVC/DLCO (predicted \% ratio) & $1.2 \pm 0.48$ & $1.34(3.76,0.69)$ & $1.1 \pm 0.38$ & $1.2(1.67,0.75)$ & 0.81 \\
\hline 6 MWT (meter) & $471 \pm 105$ & $463(720,180)$ & $408 \pm 92$ & $432(512,288)$ & 0.26 \\
\hline Before6 MWT sat. (\%) & $96 \pm 2$ & $97(100,90)$ & $93 \pm 2.7$ & $93(98,91)$ & 0.02 \\
\hline After 6 MWT sat. (\%) & $94 \pm 5$ & $96(100,71)$ & $83 \pm 10$ & $82(97,70)$ & 0.01 \\
\hline Borg (Before 6 MWT) & $0.5 \pm 0.8$ & $0(4,0)$ & $0.4 \pm 0.9$ & $0(2,0)$ & 0.52 \\
\hline Borg (After 6 MWT) & $1.45 \pm 1.7$ & $1(0,7)$ & $3.3 \pm 3$ & $4(0,7)$ & 0.17 \\
\hline Sat. difference $(\%)$ & $-2 \pm 4.2$ & $1(-24,-1)$ & $-10 \pm 8$ & $-12(-22,-1)$ & 0.007 \\
\hline Borg difference & $0.9 \pm 1.2$ & $0(0,4)$ & $3 \pm 3$ & $3(0.7)$ & 0.059 \\
\hline pH & $7.42 \pm 14$ & $7.43(7.53,7.37)$ & $7.40 \pm 0$ & $7.40(7.44,7.38)$ & 0.14 \\
\hline $\mathrm{PaCO}_{2}(\mathrm{mmHg})$ & $34 \pm 3.3$ & $35(41,26)$ & $37 \pm 5$ & $39(42,28)$ & 0.10 \\
\hline $\mathrm{PaO}_{2}(\mathrm{mmHg})$ & $75 \pm 9$ & $78(89,55)$ & $67 \pm 16$ & $68(91,45)$ & 0.09 \\
\hline
\end{tabular}


In our study all the patients had pulmonary involvement and the most common symptom was dyspnea in accordance with literature. $30 \%-50 \%$ of patients with sarcoidosis have limited FEV1 and expiratory flow rates [15] and patients usually have restrictive pathology [16]. However obstructive pathology is rarely seen alone, it is usually combined with restriction [17]. However, mean value of FEV1, FVC, DLCO and DLCO/VA predicted $\%$ were normal, 18 patients (36\%) had FEV1 $<80 \%$ and $14(28 \%)$ had FVC $<80 \%$ of predicted; $4(8 \%)$ had mixed pathology in this study. $15 \%-50 \%$ of the patients with sarcoidosis has limited DLCO [17]; in this study this percentage was $42 \%$.

6 MWT is an exercise test that reflects functional capacity in lung diseases such as chronic obstructive lung disease, idiopatic pulmonary fibrosis and PH. The studies about 6 MWT in sarcoidosis are quite limited. In a study, the mean 6 MWT distance of 142 patients with sarcoidosis was found to be $396 \mathrm{~m}$, and $51 \%$ of them walked under $400 \mathrm{~m}$, and $22 \%$ under $300 \mathrm{~m}$. They also reported significant correlation between 6 MWT and S. George respiratory questionnaire, FVC, oxygen saturation [18]. On the other hand, the mean distance of 6 MWT was $464 \mathrm{~m}$ in our study as 10 patients $(20 \%)$ walked under $400 \mathrm{~m}$, and $3(6 \%)$ walked under $300 \mathrm{~m}$. More distance walked in our study might be due to the presence of left ventricular dysfunction in 10 patients and $\mathrm{PH}$ in 14 patients with low 6 MWT score in the study by Baughman et al., despite they did not find any correlation between 6 MWT and cardiac status. In another study, 6 MWT was found to be statistically significantly related to FVC, FEV1, BORG and saturation after test in 26 patients with sarcoidosis [19]. Similarly, in our study, we found significant correlation between $6 \mathrm{MWT}$ and $\mathrm{FVC} \%$, FEV1\%, DLCO $\%, \mathrm{PaO}_{2}$. But the most significant correlation was found between 6 MWT and age. In other studies it was shown that 6 MWT distance decreased while skeletal muscle strength decreased [20]. Another the reason for the significant relationship between 6 MWT and age, decreased skeletal muscle strength with aging can reflects distance independently age effect. In our study women walked shorter than man as in Baughman's study.

Incidance of sarcoidosis associated $\mathrm{PH}$ is reported $10 \%, 70 \%$ in patients who are listed for transplantation [2]-[4]. Mean value of systolic PAP was $29 \pm 9.5 \mathrm{mmHg}$ in our study and five patients (10\%) diagnosed PH possible, likely by echo. Group 2 was composed of female. It may be due to majority of female in the study. Although it was shown that PH was more frequent in female in USA, in Japan were found to be more common in males [21].

In our study it was found that two patients (40\%) were stage 2; three (60\%) were stage 4 in group 2 . In recent studies were shown that PH could occur absence of fibrosis but the most of PH patients were stage 3-4 [21]. Likely, in other studies [22] [23] prevalence of PH was associated with stage 4. It was thought that the hypothesis that pulmonary vessel damage due to pulmonary fibrosis causes PH had been supported [22]. In our study, there was no correlation between PAP and stage. It may be due to limited PH patients.

In another study, $\mathrm{PH}$, due to fibrosis, was found that it occurred longer period and mean value of mean PAP was $40.1 \mathrm{mmHg}$. But, $\mathrm{PH}$, not associated with fibrosis, occurred earlier than other patients with PH due to fibrosis and mean value of mean PAP was to be found $51.7 \mathrm{mmHg}$. Even in stage 4, no correlation between PAP and lung volumes or arterial oxygen tensions, suggesting other mechanisms for $\mathrm{PH}$ in addition pulmonary destruction and hypoxemia [24]. Also, in our study no significant correlation was found between PAP and disease duration, stage, $\mathrm{PO}_{2}$. Limited number of patients can occur this result as well as different mechanisms.

There are different results about correlation and relationship between PFT values and PH. In our study predicted\% of FVC, FEV1 and MMF were significant lower in group 2 and significant correlation was found between PAP and FEV1\%, MMF\%. In some studies patients with PH, were advanced stage, had lower saturation and the predicted\% of VC, FVC, FEV1, FRC, TLC, FEF 25 - 75, DLCO [24] [25] [27]. In Sulica's study, it was shown that patients who had been stage 4 and with $\mathrm{PH}$, had had lover MMF\% and it was found that in the presence of fibrosis, decreased MMF\% value had been related to exercise induced PAP. It was suggested that decreased MMF\% and occurrence of $\mathrm{PH}$ could have been related to granulomatous inflammation and fibrosis in small airway and small and medium-sized vessels [23].

$\mathrm{DLCO}<60 \%$ has been reported to be related to an increase in prevalence of underlying $\mathrm{PH}$ [26]. In our study there was no significant difference between groups, however mean value of DLCO $<80 \%$ in group 2, $>80 \%$ in group 1. Although FVC/DLCO predicted\% ratio in screening for PH has never been tested in sarcoidosis, several authors have postulated that a high ratio $(>1.4-1.5)$, reflecting a disproportionately reduced DLCO with/ without the oxygen saturation, may be a better screening tool for PH in intestinal lung diseases than any other PFT [27] [28]. In our study the mean value of FVC/ DLCO predicted\% ratio was $1.2 \pm 0.48$ in group 1, $1.1 \pm$ 0.38 in group 2. No significant difference was found between groups. This ratio showed proportional decrement 
in $\mathrm{FVC} \%$ and $\mathrm{DLCO} \%$. Low ratio was due to limited number of $\mathrm{PH}$ patients.

In Baughman's study PH was associated with a significantly shorter 6 MWT distance, but no correlation was found between the systolic or mean pulmonary artery pressure, and the 6 MWT [18]. In our study there was no significant both difference in comprasion of the groups and in correlation between PAP and 6 WMT. This result can related to limited number of $\mathrm{PH}$ or, our patients can have limited factors that affect 6 WMT such as systemic disorders or sarcoidosis involvement. The multisystem nature of sarcoidosis invokes other reasons for a lower distance attained during the 6 MWT. Therefore it is possible that the distance component is more useful to evaluate response to therapy, rather than as a screening tool for PH [12] [18] [29].

Exercise desaturation is another important indicator for PH. Patients who desaturate below $90 \%$ with exercise are 12-fold more likely to have associated PH [26] [18]. In our study, both group had saturation $>90 \%$ at rest, but in group 2 desaturated below $90 \%$ after 6 MWT and it was also statically significant.

In our study the most important limitation was limited patients' number and big difference in number of patients between groups.

\section{Conclusion}

$\mathrm{PH}$ is an important complication of sarcoidosis. In all stages, patients who have longer disease duration and abnormal pulmonary function tests, should be examined about PH. Patients should undergo 6 MWT and presence of desaturation after 6 MWT, even without hypoxemia, should be looked after.

\section{References}

[1] Musellim, B., Kumbasar, Ö.Ö. and Ongen, G. (2009) Epidemiological Features of Turkish Patients with Sarcoidosis. Respiratory Medicine, 103, 907-912. http://dx.doi.org/10.1016/j.rmed.2008.12.011

[2] Nunes, H., Humbert, M., Capron, F., Brauner, M., Sitbon, O., Battesti, J.P., Simonneau, G. and Valeyre, D. (2006) Pulmonary Hypertension Associated with Sarcoidosis: Mechanisms, Hemodynamic and Prognosis. Thorax, 61, 68-74. http://dx.doi.org/10.1136/thx.2005.042838

[3] Baughman, R.P., Engel, P.J., Taylor, L. and Lower, E.E. (2010) Survival in Sarcoidosis Associated Pulmonary Hypertension: The Importance of Hemodynamic Evaluation. Chest, 138, 1078-1085. http://dx.doi.org/10.1378/chest.09-2002

[4] Bourbonnais, J.M. and Samavati, L. (2008) Clinical Predictors of Pulmonary Hypertension in Sarcoidosis. European Respiratory Journal, 32, 296-302. http://dx.doi.org/10.1183/09031936.00175907

[5] Brauner, M., Grenier, P. and Mompoint, D. (1989) Pulmonary Sarcoidosis: Evaluation with High-Resolution CT. Radiology, 172, 467-471. http://dx.doi.org/10.1148/radiology.172.2.2748828

[6] Huang, C., Heurich, A., Rosen, Y., Moon, S. and Lyons, H.A. (1979) Pulmonary Sarcoidosis: Roentgenographic, Functional and Pathologic Correlations. Respiration, 37, 337-345. http://dx.doi.org/10.1159/000194046

[7] Sharma, O. (1994) Pulmonary Sarcoidosis: Radiographic Features. In: James, D.O., Ed., Sarcoidosis and Other Granulomatous Disorders, Marcel Dekker, New York, 213-245.

[8] Fraser, R.G. and Pare, J.A.P. (1979) Diagnosis of Diseases of the Chest. W.B. Saunders Company, Philadelphia, 16591689.

[9] Rizzato, G., Pezzano, A., Sala, G., Merlini, R., Ladelli, L., Tansini, G., et al. (1983) Right Heart Impairment in Sarcoidosis: Hemodynamic and Echocardiographic Study. European Journal of Respiratory Diseases, 64, 121-128.

[10] ATS Statement (2002) Guidelines for the Six-Minute Walk Test. American Journal of Respiratory and Critical Care Medicine, 166, 111-117. http://dx.doi.org/10.1164/ajrccm.166.1.at1102

[11] Arcasoy, S.M., Christie, J.D., Ferrari, V.A., Sutton, M.S., Zisman, D.A., Blumenthal, N.P., Pochettino, A. and Kotloff, R.M. (2003) Echocardiographic Assessment of Pulmonary Hypertension in Patients with Advanced Lung Disease. American Journal of Respiratory and Critical Care Medicine, 167, 735-740. http://dx.doi.org/10.1164/rccm.200210-11300C

[12] Palmero, V. and Sulica, R. (2010) Sarcoidosis-Associated Pulmonary Hypertension: Assessment and Management. Seminars in Respiratory and Critical Care Medicine, 31, 494-500. http://dx.doi.org/10.1055/s-0030-1262217

[13] European Society of Cardiology (ESC) and European Respiratory Society (ERS) (2009) Guidelines for the Diagnosis and Treatment of Pulmonary Hypertension. European Heart Journal, 30, 2493-2537. http://dx.doi.org/10.1093/eurheartj/ehp297

[14] Judson, A.M. (2014) Pulmonary Sarcoidosis: A Guide for the Practicing Clinician. Humana Press, New York, 11-29.

[15] Mihailovic-Vucinic, V., Zugic, V. and Videnovic-Ivanov, J. (2003) New Observations on Pulmonary Function 
Changes in Sarcoidosis. Current Opinion in Pulmonary Medicine, 9, 436-441. http://dx.doi.org/10.1097/00063198-200309000-00017

[16] Lynch III, J.P., Kazerooni, E.A. and Gay, S.E. (1997) Pulmonary Sarcoidosis. Clinics in Chest Medicine, 18, 755-785. http://dx.doi.org/10.1016/S0272-5231(05)70417-2

[17] Harrison, B.D., Shaylor, J.M. and Stokes, T.C. (1991) Airflow Limitation in Sarciodosis. Respiratory Medicine, 85, 5964. http://dx.doi.org/10.1016/S0954-6111(06)80211-8

[18] Baughman, R.B., Sparkman, B.K. and Lower, E.E. (2007) Assessment in Sarcoidosis Six-Minute Walk Test and Health Status. Chest, 132, 207-213. http://dx.doi.org/10.1378/chest.06-2822

[19] Alhamad, E.H. (2009) The Six-Minute Walk Test in Patients with Pulmonary Sarcoidosis. Annals of Thoracic Medicine, 4, 60-63.

[20] Rasekaba, T., Lee, A.L., Naughton, M.T., Williams, T.J. and Holland, A.E. (2009) The Six-Minute Walk Test: A Useful Metric for the Cardiopulmonary Patient. Internal Medicine Journal, 39, 495-501. http://dx.doi.org/10.1111/j.1445-5994.2008.01880.x

[21] Guzman, D.E., Farver, C., Parambil, J. and Culver, D.A. (2008) Pulmonary Hypertension Caused by Sarcoidosis. Clinics in Chest Medicine, 29, 549-563. http://dx.doi.org/10.1016/j.ccm.2008.03.010

[22] Handa, T., Sonoko, N. and Shinji, M. (2006) Incidence of Pulmonary Hypertension and Its Clinical Relevance in Patients with Sarcoidosis. Chest, 129, 1246-1252. http://dx.doi.org/10.1378/chest.129.5.1246

[23] Sulica, R., Teirstein, A.S., Kakarla, S., Nemani, N., Behnegar, A. and Padilla, M.L. (2005) Distinctive Clinical, Radiographic, and Functional Characteristics of Patients With Sarcoidosis-Related Pulmonary Hypertension. Chest, 128, 1483-1489. http://dx.doi.org/10.1378/chest.128.3.1483

[24] Nunes, H., Humbert, M. and Capron, F. (2006) Pulmonary Hypertension Associated with Sarcoidosis: Mechanisms, Haemodynamics and Prognosis. Thorax, 61, 68-74. http://dx.doi.org/10.1136/thx.2005.042838

[25] Shorr, A.F., Helman, D.L., Davies, D.B. and Nathan, S.D. (2005) Pulmonary Hypertension in Advanced Sarcoidosis: Epidemiology and Clinical Characteristics. European Respiratory Journal, 25, 783-788. http://dx.doi.org/10.1183/09031936.05.00083404

[26] Bourbonnais, J.M. and Samavati, L. (2008) Clinical Predictors of Pulmonary Hypertension in Sarcoidosis. European Respiratory Journal, 32, 296-302. http://dx.doi.org/10.1183/09031936.00175907

[27] Steen, V.D., Graham, G., Conte, C., Owens, G. and Medsger Jr., T.A. (1992) Isolated Diffusing Capacity Reduction in Systemic Sclerosis. Arthritis \& Rheumatology, 35, 765-770. http://dx.doi.org/10.1002/art.1780350709

[28] Nathan, S.D., Shlobin, O.A., Ahmad, S., Urbanek, S. and Barnett, S.D. (2007) Pulmonary Hypertension and Pulmonary Function Testing in Idiopathic Pulmonary Fibrosis. Chest, 131, 657-663. http://dx.doi.org/10.1378/chest.06-2485

[29] Rosen, Y., Moon, S., Huang, C.T., Gourin, A. and Lyons, H.A. (1977) Granulomatous Pulmonary Agilities in Sarcoidosis. Archives of Pathology \& Laboratory Medicine, 101, 170-174. 